\title{
Modelo de optimización para la ubicación de Access Point en redes WLAN
}

\section{Optimization model for the location of Access Point in WLAN networks}

INVESTIGACIÓN E INNOVACION en INGENERIAS

ISSN2344-8652

\section{OPEN A ACCESS}

Recibido: 08/01/2021

Aceptado: 13/04/2021

Publicado: 15/06/2021

Correspondencia de autores: martha.contreras@unad.edu.co

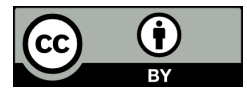

Copyrith 2020

by Investigación e Innovación en Ingenierías
Resumen

Objetivo: Proponer un modelo de optimización para la ubicación de AP en redes WLAN en ambientes Indoor. Metodología: Para su desarrollo se plantearon algoritmos que permiten estimar la probabilidad de corte, el área de cobertura y las coordenadas de ubicación de los AP en función de las dimensiones del escenario, la frecuencia y las condiciones del entorno, soportadas en el modelo de propagación Log-Normal Shadowing Path Loss. Resultados y Conclusiones: Con base en los resultados obtenidos se pudo evidenciar que el modelo propuesto arrojó excelentes resultados al determinar el área de cobertura, la probabilidad de corte para un $80 \%$, la ubicación geográfica de los AP acorde con los parámetros establecidos en cada uno de los escenarios, motivo por el cual se recomendaría el uso del modelo en futuros trabajos de investigación relacionados con la temática objeto de estudio.

Palabras clave: WLAN, optimización, ubicación de AP, área de cobertura, probabilidad de corte.

Abstract

Objective: To propose an optimization model for the location of AP in WLAN networks in Indoor environments. Methodology: For its development algorithms were proposed that allow estimating the probability of cut, coverage area and location coordinates of the APs based on the dimensions of the scenario, frequency and environmental conditions, supported in the model propagation LogNormal Shadowing Path Loss. Results and Conclusions: Based on the results obtained, it was possible to show that the proposed model showed excellent results when determining the coverage area, the cut probability for $80 \%$, the geographical location of the PAs according to the parameters established in each of the scenarios, which is why the use of the model in future research works related to the subject under study would be recommended.

Keywords: WLAN, optimization, AP location, coverage area, cut probability

Como citar (IEEE): M. Contreras-Higuera., J. Vesga-Ferreira., J. Vesga-Barrera “Modelo de optimización para la ubicación de Access Point en redes WLAN", Investigación e Innovación en Ingenierías, vol. 9, n¹, 180-195, 2021. DOI: https://doi.org/10.17081/ invinno.9.1.4203 


\section{Introducción}

A lo largo de los últimos años la demanda de redes inalámbricas por parte de los usuarios ha venido en aumento, debido a los beneficios de movilidad y bajo costo de implementación [1]. Dentro de la gama de redes inalámbricas, están las denominadas WLAN (Wireless Local Area Network), las cuales se han estado utilizando en lugares cotidianos tales como: hogares, oficinas, instituciones educativas, entre otros [2]. Este tipo de redes están conformadas principalmente por el uso de dispositivos concentradores denominados Access Point (AP), los cuales permiten que los usuarios se puedan conectar de manera inalámbrica a la red, cumpliendo la misma función que un Switch en una red cableada [3]. Sin embargo, pese a que los AP permiten establecer conexiones de manera inalámbrica, la distancia a la que debe estar el usuario del AP es bastante reducida (inferior a 100m), debido a la potencia de la señal de transmisión y los obstáculos que la señal pueda encontrar en el entorno para alcanzar al usuario [4]. Es por tal razón que, debido a las necesidades de contar con una conexión inalámbrica por parte de los usuarios, la proliferación de AP sea cada vez mayor.

El aumento en la densidad de AP en redes WLAN ha comenzado a revelar los efectos negativos y las deficiencias existentes en el estándar IEEE 802.11n [5]. Uno de sus principales factores de éxito, el uso de bandas libres, es al mismo tiempo uno de sus principales inconvenientes, debido a que estas frecuencias son de libre acceso y de uso compartido entre diversos usuarios, dispositivos y tecnologías inalámbricas tales como Bluetooth, ZigBee, Zwave e incluso WiFi (Wireless Fidelity), afectando considerablemente el rendimiento de la red debido a factores de interferencia, ubicación de los AP y al manejo ineficiente del espectro electromagnético [6,7]. El problema de diseño y optimización de las redes inalámbricas ha sido abordado desde hace mucho tiempo, promoviendo un gran número de investigaciones $[8,9,10,11]$. Sin embargo, aunque se han realizado diversos trabajos relacionados con la optimización de recursos en redes WLAN, son muy pocos los trabajos que han considerado involucrar dentro de sus investigaciones estrategias para la optimización de la ubicación geográfica de los AP, aspecto que juega un papel fundamental en éste proceso $[12,13]$.

En vista de lo anterior, en el presente artículo se propone un modelo que permite optimizar el número de APs requerido y su ubicación geográfica al interior de la construcción, teniendo en cuenta los niveles de potencia, modelos de propagación, patrones de interferencia y radios de cobertura en la banda de 2.4 $\mathrm{GHz}$ y $5 \mathrm{GHz}$. El modelo es planteado como un problema de programación no lineal, ofreciendo una baja complejidad computacional y temporal para su uso e implementación.

\section{Metodología}

\section{A. Área de Cobertura y Probabilidad de Corte}

La atenuación por sombra tiene una gran influencia en los procesos de diseño de sistemas inalámbricos [14]. Debido al comportamiento aleatorio de la señal de potencia recibida es difícil conocer con precisión el alcance máximo acorde con la sensibilidad en calidad de potencia del Receptor [15,12]. Ante esta situación es necesario estimar la "Probabilidad de Corte" y el "Área de cobertura", conceptos que son de vital importancia en los procesos de diseño de redes inalámbricas. 
Se denomina "Probabilidad de Corte $\left(P_{\text {cut }}\right)$ " a aquella probabilidad en la cual la potencia recibida Pr a una distancia d del transmisor quede por debajo del umbral de recepción Pmin [16].

$$
P_{\text {cut }}\left(P_{\text {min }}, d\right)=\operatorname{Prob}\left\{P_{r}(d)<P_{\min }\right\}
$$

Es posible descomponer la potencia recibida en una potencia promedio y un término de atenuación por sombra.

$$
P_{r}(d)=\bar{P}_{r}(d)-\varphi_{d B}
$$

Podemos escribir la probabilidad de corte como:

$$
P_{\text {cut }}\left(P_{\text {min }}, d\right)=\operatorname{Prob}\left\{\varphi_{d B}>\overline{\Delta P}\left(P_{\text {min }}, d\right)\right\}
$$

Teniendo en cuenta la naturaleza Log-Normal

$$
P_{c u t}\left(P_{\text {min }}, d\right)=Q\left(\frac{\overline{\Delta P}\left(P_{\text {min }}, d\right)}{\sigma_{\varphi_{d B}}}\right)=Q\left(\frac{\overline{P_{r}}(d)-P_{\text {min }}}{\sigma_{\varphi_{d B}}}\right)
$$

Donde $Q(z)$ es la probabilidad de que una variable $N(0,1)$ sea mayor que Z, es decir:

$$
Q(z)=\int_{z}^{\infty} \frac{1}{\sqrt{2 \pi}} e^{x^{2} / 2} d x=\frac{1}{2} \operatorname{erfc}\left(\frac{z}{\sqrt{2}}\right)
$$

Se denomina "Área de cobertura" al promedio del porcentaje de superficie en el cual se espera recibir un nivel de señal igual o superior al umbral de detección $P_{\min }$ [17]. En un modelo que no considere la atenuación por sombra y se considera que el radio de la célula (supuesta circular) sea igual a la distancia en la cual la potencia promedio recibida $P_{r}=P_{\text {min }}$, el área de cobertura será del 100\% [18]. Sin embargo, al considerar la atenuación por sombra es necesario tener en cuenta lo siguiente:

$$
1-P_{\text {cut }}\left(P_{\text {min }}, d\right)=Q\left(\frac{-\overline{\Delta P}\left(P_{\text {min }}, d\right)}{\sigma_{\varphi_{d B}}}\right)
$$

La expresión que permite calcular el área de cobertura en una red inalámbrica bajo el modelo log-normal es la siguiente:

$$
\begin{gathered}
C\left(P_{\text {min }}, R\right)=\frac{1}{\pi R^{2}} \int_{0}^{R} 2 \pi r\left(1-P_{\text {cut }}\left(P_{\text {min }}, r\right)\right) d r \\
C\left(P_{\text {min }}, R\right)=\frac{2}{R^{2}} \int_{0}^{R} r Q\left(\frac{-\overline{\Delta P}\left(P_{\text {min }}, r\right)}{\sigma_{\varphi_{d B}}}\right) d r
\end{gathered}
$$

Donde ${ }^{r}$ corresponde al radio de cobertura. Teniendo en cuenta que:

$$
\overline{P_{r}}=P_{t}+K-10 \log \left[d / d_{0}\right]
$$


Es posible expresar el margen de potencia en función de su valor en frontera de la célula.

$$
\overline{\Delta P}\left(P_{\min }, r\right)=\overline{\Delta P}\left(P_{\min }, R\right)-10 \cdot \gamma \cdot \log [r / R]
$$

Reemplazando $\overline{\Delta P}\left(P_{\min }, r\right)$ en $C\left(P_{\min }, R\right)$ :

$$
\begin{gathered}
C\left(P_{\text {min }}, R\right)=\frac{2}{R^{2}} \int_{0}^{R} r Q\left(\frac{-\overline{\Delta P}\left(P_{\text {min }}, R\right)+10 \cdot \gamma \cdot \log \left[\frac{r}{R}\right]}{\sigma_{\varphi_{d B}}}\right) d r \\
C\left(P_{\text {min }}, R\right)=\frac{2}{R^{2}} \int_{0}^{R} r Q\left[a+b \cdot \ln \left(\frac{r}{R}\right)\right] d r
\end{gathered}
$$

Con,

$$
a=\frac{\overline{\Delta P}\left(P_{\min }, R\right)}{\sigma_{\varphi_{d B}}} \quad b=\frac{10 \cdot \gamma \cdot \log (e)}{\sigma_{\varphi_{d B}}}
$$

La expresión resultante para estimar el área de cobertura es:

$$
C(a, b)=Q(a)+e^{\frac{2-2 a b}{b^{2}}} Q\left[\frac{2-a b}{b}\right]
$$

En términos de diseño, para cada célula se considera a $P_{\min }=\bar{P}_{r}(R)$ de tal forma que el margen en la frontera $\overline{\Delta P}\left(P_{\min }, R\right)$ sea nulo, en tal caso $a=0$ y el área de cobertura dependería solamente de $b$, el cual es proporcional a $\frac{\gamma}{\sigma \varphi_{d B}}$. Reemplazando el valor de $a=0$ en la expresión anterior, el resultado sería:

$$
C_{(b)}=\frac{1}{2}+e^{2 / b^{2}} Q\left(\frac{2}{b}\right)
$$

A continuación, se presenta el pseudocódigo que permite la estimación del radio de cobertura y la probabilidad de corte para la banda de 2.4 y $5 \mathrm{GHz}$, denominada PrCut.

Pseudocódigo para estimación del radio de cobertura y probabilidad de corte

// Tipo: Indicador Espacio libre/Obstáculos;

// r: Radio de cobertura en metros

// Pmin: Umbral de detección en dBm

// Pt: Potencia de Tx en dBm

// PC : Probabilidad de Corte estimada en porcentaje

// Pcut : Probabilidad de Corte real

// C: Área de Cobertura en Porcentaje 
función [ r,Pr,Pcut,C ] = Prob_Corte24G(Pt,Tipo,Pmin,PC)

// Condiciones Iniciales de iteración

1. Pcorte=0; // Probabilidad Inicial

2. $r=0 ; \quad / /$ Distancia Inicial

3. Mientras Pcorte<PC hacer

a. $r=r+1$

b. $\mathrm{Pr}=\mathrm{Pt}+(\mathrm{K}-10 *$ gamma* $\log 10(\mathrm{r})) ;$ // Potencia recibida a una distancia $\mathrm{r}$

// Probabilidad de corte

c. Pcut $=100 *(0.5 * \operatorname{erfc}((\operatorname{Pr}-\mathrm{Pmin}) /($ Sigma_dB*sqrt(2))));

// Área de cobertura

d. $a=(P m i n-P r) / S i g m a \_d B ;$

e. $b=10 *$ gamma* $\log 10(\exp (1)) /$ Sigma_dB;

f. $C=100 *\left(0.5^{*} \operatorname{erfc}(a / \operatorname{sqrt}(2))+\left(\exp \left(\left(2-2^{*} a * b\right) / b^{\wedge} 2\right) * 0.5^{*} \operatorname{erfc}\left(\left(2-a^{*} b\right) /(b * \operatorname{sqrt}(2))\right)\right)\right)$;

Repetir

Fin de función

Fuente: Elaboración propia

En la Tabla 1 se registran los parámetros correspondientes al modelo de atenuación por sombra en función del entorno y banda de frecuencia respectiva.

Tabla 1. Parámetros para estimación del radio de cobertura y probabilidad de corte [15]

\begin{tabular}{|c|c|c|c|c|}
\hline \multirow{2}{*}{ Frecuencia } & Entorno & Gamma & Sigma [dB] & 5,54 \\
\hline \multirow{2}{*}{$2.4 \mathrm{GHz}$} & Espacio libre & 2,216 & 4,67 & $-49,95$ \\
\cline { 2 - 5 } & Con Obstáculos & 3,298 & $-48,89$ & $-51,877$ \\
\hline \multirow{2}{*}{$5 \mathrm{GHz}$} & Espacio libre & 2,66 & 5,697 & $-66,29$ \\
\hline
\end{tabular}

Fuente: Elaboración propia

En la Figura 1 se presentan las curvas que describen el área de cobertura para escenarios en espacio libre y entornos con obstáculos para las bandas de $2.4 \mathrm{GHz}$ y $5 \mathrm{GHz}$. En ellas se puede apreciar que la banda de $2.4 \mathrm{GHz}$ ofrece áreas de cobertura superiores a la banda de $5 \mathrm{GHz}$ teniendo en cuenta la misma distancia de referencia, lo cual permitirá no solo estimar los radios de cobertura adecuados para cada caso, sino que además facilitará la estimación de los rangos de alcance de la señal en entornos con obstáculos como en espacio libre, a fin de garantizar un adecuado servicio a cada uno de los usuarios y evitando la presencia de zonas de silencio que puedan llegar a presentarse en un momento dado. 
Figura 1. Área de cobertura en función del entorno y la banda de frecuencia

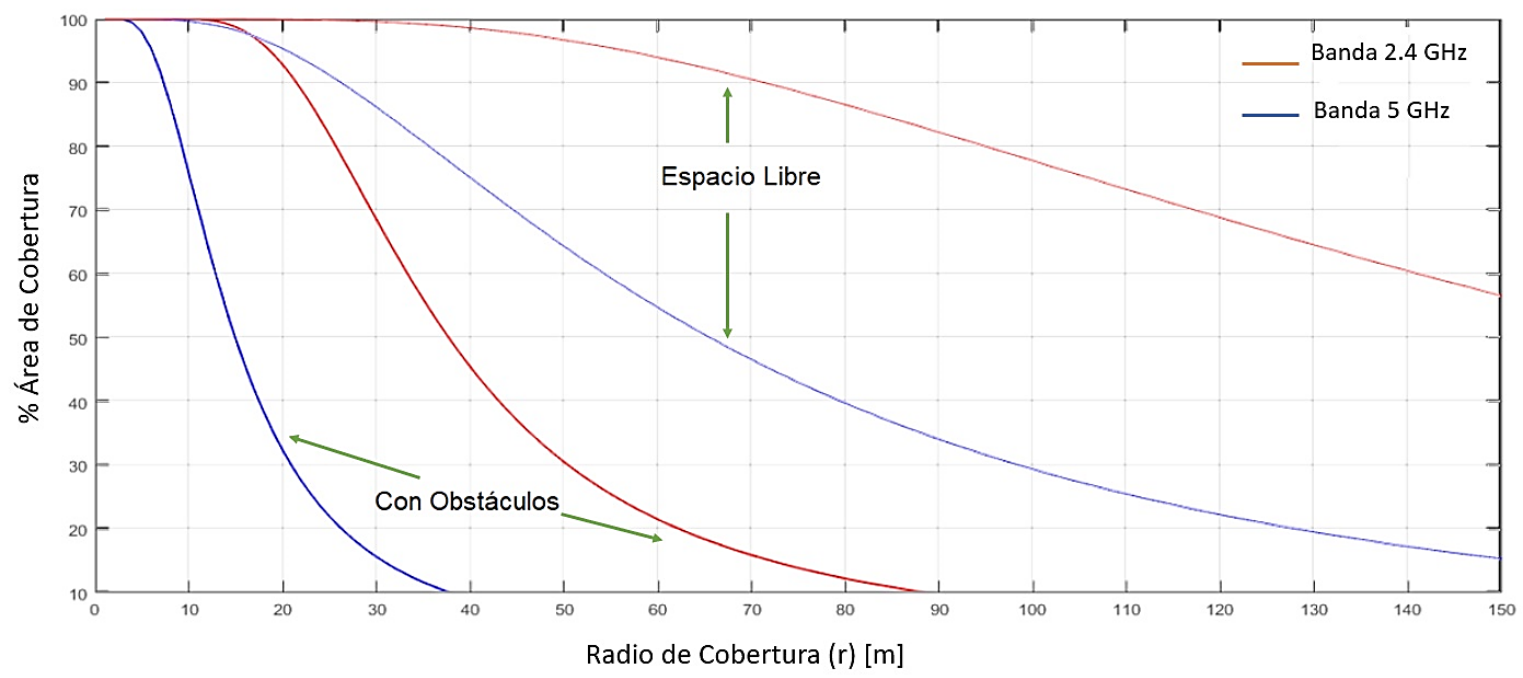

Fuente: Elaboración propia

Figura 2. Probabilidad de corte en función del entorno y la banda de frecuencia

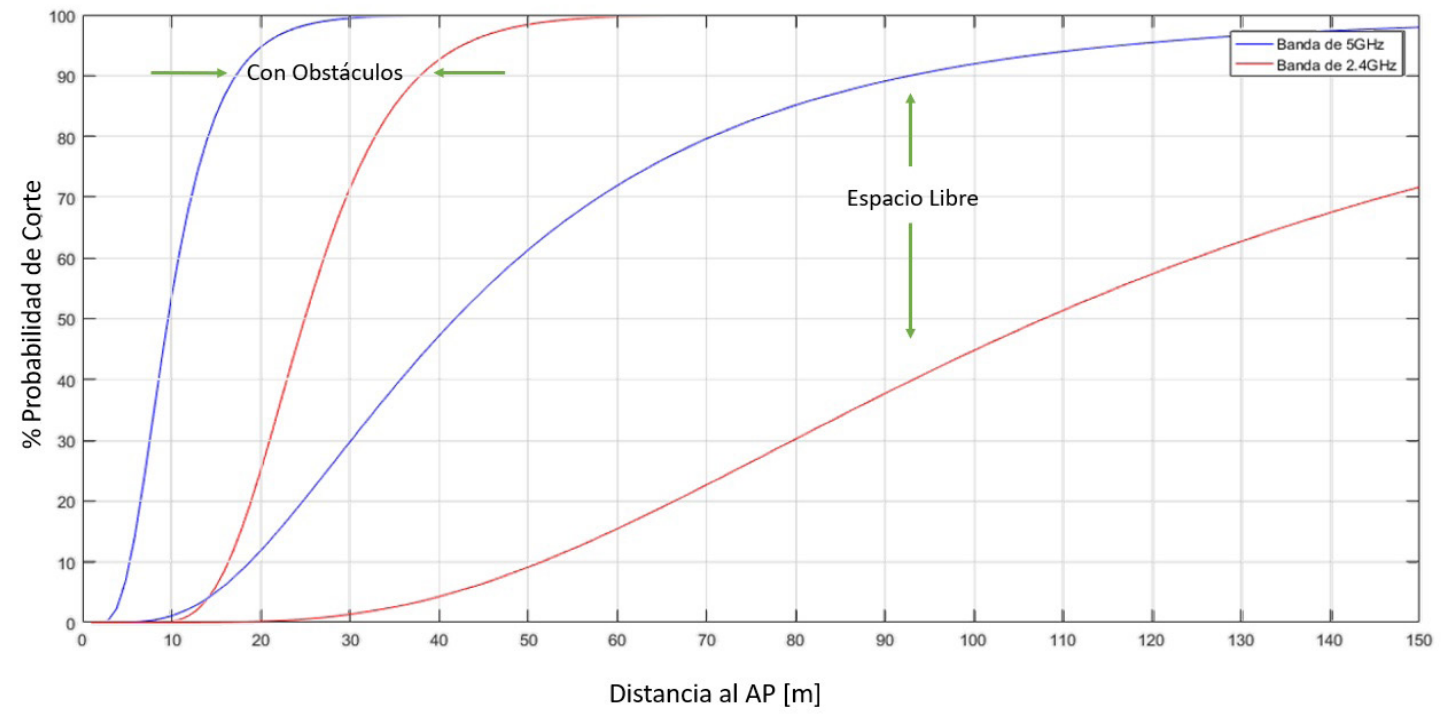

Fuente: Elaboración propia

En la Figura 2 se presentan las curvas que describen la probabilidad de corte para escenarios en espacio libre y entornos con obstáculos en función de las bandas de $2.4 \mathrm{GHz}$ y $5 \mathrm{GHz}$. En ellas se puede apreciar que la banda de $5 \mathrm{GHz}$ ofrece una probabilidad de corte superiores a la banda de $2.4 \mathrm{GHz}$ teniendo en cuenta la misma distancia de referencia y con ello optimizar los procesos de diseño de redes inalámbricas al identificar con mayor claridad los límites de conectividad entre celdas, radios de cobertura adecuados para cada caso a fin de garantizar un adecuado servicio a cada uno de los usuarios. 
Acorde con el modelo de atenuación por sombra que fue considerado, la potencia de recepción en la banda de $5 \mathrm{GHz}$ ofrece niveles de atenuación mayores que la banda de $2.4 \mathrm{GHz}$ a la misma distancia de referencia. Este aspecto es muy importante debido a que a través de las expresiones consideradas para el modelo de atenuación por sombra permitirán estimar los posibles radios de cobertura de la mano con la probabilidad de corte y el área de cobertura, en función de los niveles de sensibilidad de los dispositivos receptores, que en la mayoría de los casos para dispositivos móviles se encuentran con valores cercanos a los -70dBm y para PC y Laptop a los $-80 \mathrm{dBm}$.

\section{B. Metodología para la optimización de la ubicación geográfica de los AP}

Como parte del trabajo de investigación realizado se diseñó una metodología para la optimización de la ubicación geográfica de los AP al interior de una construcción. Para ello se deben realizar los siguientes pasos:

Paso 1. Estimación del radio promedio de cobertura para cada una de las bandas de frecuencia, el cual puede ser calculado en función de la Probabilidad de Corte, la banda de frecuencia, el tipo de cobertura (espacio libre o con obstáculos) y la sensibilidad de recepción. Para ello se hace uso de la función PrCut (Pt,Tipo,Pmin,Pc,gamma,Sigma,K) descritas anteriormente. En la Tabla 2 se presentan los resultados obtenidos acorde con los parámetros de entrada requeridos por cada una de las funciones.

Tabla 2. Estimación del radio de cobertura, probabilidad de corte y probabilidad de cobertura para valores específicos de diseño en las bandas de $2.4 \mathrm{GHz}$ y $5 \mathrm{GHz}$

\begin{tabular}{|c|c|c|c|c|}
\hline Parámetros de Diseño & \multicolumn{2}{|c|}{ Banda 2.4GHz Banda 5Gz } \\
\hline Potencia de Transmisión (Pt) [dBm] & 26 & 26 & 25 & 25 \\
\hline Tipo & Espacio Libre & Obstáculos & Espacio Libre & Obstáculos \\
\hline Sensibilidad Pmin [dBm] & -70 & -70 & -80 & -80 \\
\hline Prob. de corte estimada Pc[\%] & 15 & 15 & 15 & 15 \\
\hline Prob. de corte real (Pcut) [\%] & 15,05 & 18,41 & 15,12 & 15,65 \\
\hline Radio de Cobertura (r) [m] & 66 & 20 & 53 & $-74,66$ \\
\hline Potencia de Recepción (Pr)[dBm] & $-64,27$ & $-65,79$ & $-72,74$ & 94,18 \\
\hline Cobertura (C) [\%] & 94,15 & 95,00 & 93,56 & \\
\hline
\end{tabular}

Fuente: Elaboración propia

Paso 2. Estimación del número de AP requeridos en cada banda de frecuencia por nivel de la edificación en función del radio promedio de cobertura obtenido en el paso 1. En la Figura 3 se presenta la estimación geométrica de parámetros de diseño tales como Lado y Semi arco de Interferencia, los cuales han sido considerados por criterio para el diseño del modelo de optimización y juegan un papel importante dentro de las restricciones que forman parte del modelo propuesto para la ubicación geográfica de los AP. 
Figura 3. Estimación geométrica de parámetros de diseño (Lado y Semi arco de Interferencia)

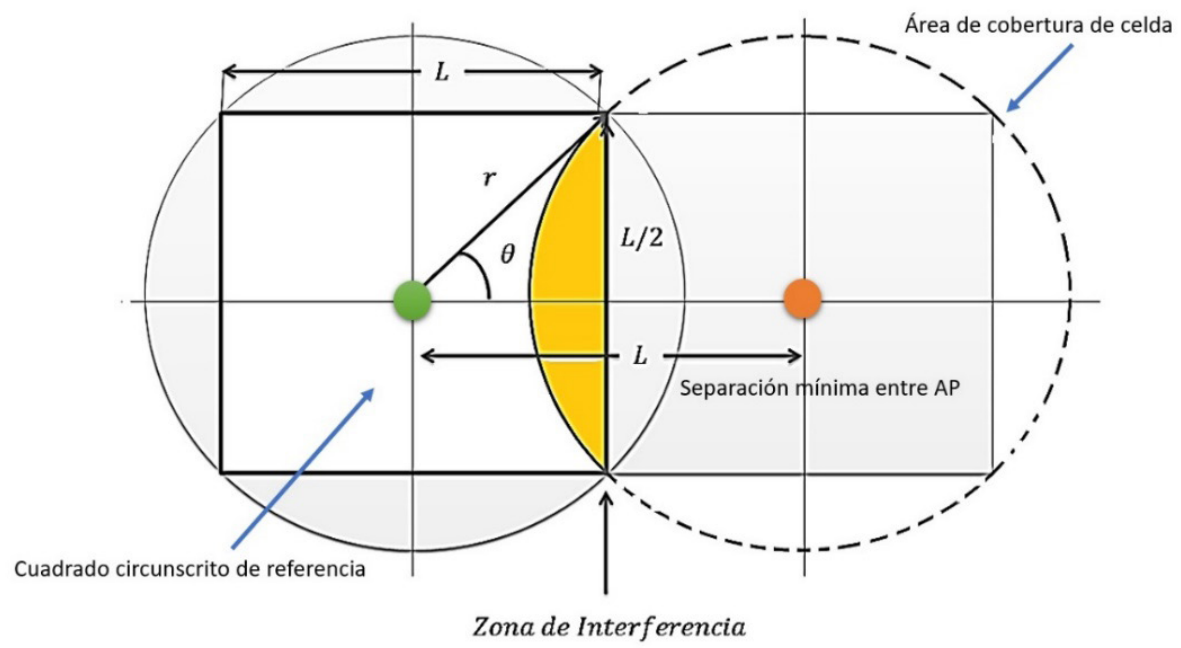

Fuente: Elaboración propia

En la Figura 3 se observa que se desea estimar el valor correspondiente al cuadrado más grande que puede ser inscrito al interior de la circunferencia de radio $r$, donde $r$ corresponde al radio de cobertura calculado en el paso anterior para cada una de las bandas de frecuencia y entornos de propagación. Teniendo en cuenta que para criterios de diseño siempre se debe considerar la peor condición, se adoptará como criterio de diseño bajo este principio que el radio de cobertura que será considerado tanto en la banda de $2.4 \mathrm{GHz}$ como en la de $5 \mathrm{GHz}$ será aquel que se obtuvo producto de calcular el radio de cobertura para entornos con obstáculos.

En vista de lo anterior y acorde con el diagrama geométrico, el valor correspondiente al Lado del cuadrado inscrito puede ser calculado de la siguiente forma:

$$
\begin{gathered}
r=\sqrt{(L / 2)^{2}+(L / 2)^{2}} \\
r=\sqrt{2 L / 4}=L / \sqrt{2} \\
L=r \cdot \sqrt{2}
\end{gathered}
$$

Por otro lado, se observa que la unión de dos cuadrados inscritos a través de un lado común permite analizar la forma en la cual dos celdas con diferente frecuencia podrían interactuar entre sí, definiendo no solo el área de cobertura establecido por la unión de las mismas, sino que además permite conocer el área de interferencia que existirá entre ellas, teniendo en cuenta una separación mínima entre AP de $L$ metros. Aspecto por el cual el factor L cumplirá un papel muy importante dentro del modelo de optimización, teniendo en cuenta que la separación entre AP deberá ser mayor e igual que $L$.

Paso 3. Para estimar el área del semi-arco de interferencia se utilizará como base el diagrama geométrico de la Figura 3. Para calcularlo se observa que se forma un ángulo $\theta$ entre el radio de cobertura y la esquina superior del cuadrado. Es muv importante tener en cuenta que el área del semi arco dependerá de la distancia entre AP y del ángulo $\theta$. Para calcular la distancia entre el $A P_{i}$ y el $A P_{j}$ ubicados en las coordenadas cartesianas $\left(X_{i}, Y_{i}\right)$ y $\left(X_{j}, Y_{j}\right)$ respectivamente, se hace uso de la siguiente expresión: 


$$
d_{i j}=\sqrt{\left(X_{i}-X_{j}\right)^{2}+\left(Y_{i}-Y_{j}\right)^{2}}
$$

El ángulo $\theta$ está dado por:

$$
\theta_{i j}=\theta\left(d_{i j}\right)=\operatorname{Acos}\left[\frac{d_{i j}}{2 r}\right]
$$

Para estimar el área de interferencia entre celdas se procede de la siguiente forma:

Sea $A_{s a}$ el área del semi arco superior

$$
A_{s a}=\frac{\theta_{i j} r^{2}}{2}
$$

Sea $A_{T}$ el área del triángulo superior

$$
A_{T}=\frac{r \cos \left(\theta_{i j}\right) \cdot r \sin \left(\theta_{i j}\right)}{2}=\frac{r^{2} \cos \left(\theta_{i j}\right) \cdot \sin \left(\theta_{i j}\right)}{2}
$$

Sea $A_{I}\left(\theta_{i j}\right)$ el área de interferencia entre celdas producto de los $A P_{i}$ y $A P_{j}$, la cual puede ser calculada de la siguiente forma:

$$
\begin{gathered}
A_{I}\left(\theta_{i j}\right)=2\left(2 A_{s a}-2 A_{T}\right)=2\left[2\left(\frac{\theta_{i j} r^{2}}{2}\right)-2\left(\frac{r^{2} \cos \left(\theta_{i j}\right) \cdot \sin \left(\theta_{i j}\right)}{2}\right)\right] \\
A_{I}\left(\theta_{i j}\right)=2\left[\theta_{i j} r^{2}-r^{2} \cos \left(\theta_{i j}\right) \cdot \sin \left(\theta_{i j}\right)\right] \\
A_{I}\left(\theta_{i j}\right)=2 r^{2}\left[\theta_{i j}-\cos \left(\theta_{i j}\right) \cdot \sin \left(\theta_{i j}\right)\right] u(\Delta d)
\end{gathered}
$$

Donde:

$$
\begin{gathered}
\Delta d=2 r-d \\
u(\Delta d)= \begin{cases}1 & \text { si } \Delta d \geq 0 \\
0 & \text { si } \Delta d<0\end{cases}
\end{gathered}
$$

Paso 4. Calcular el número de AP requerido. Para este proceso se debe calcular en primera instancia el área total del entorno $\left(\right.$ Area $\left._{E}\right)$.

$$
\text { Area }_{E}=\operatorname{Largo} * \text { Ancho }
$$

Posteriormente, se calcula el área de uno de los cuadrados inscritos en el área de cobertura de una celda.

$$
\operatorname{Area}_{C}=L * L
$$

El número de AP requeridos $(m)$ es igual al cociente entre el área del entorno y el área del cuadrado inscrito.

$$
m=\left\lceil\frac{\text { Area }_{E}}{\text { Area }_{C}}\right\rceil
$$


Paso 5. Introducir parámetros de cobertura y resolver el problema de optimización no lineal para establecer la ubicación de los AP al interior de la construcción, a fin de maximizar el área de cobertura total del sistema de celdas asociadas sobre el entorno, a través del siguiente modelo de optimización

Función Objetivo

$$
\max \left[m \pi r^{2}-\sum_{j=1}^{m} A_{I}\left(\theta_{i j}\right) \forall i, j=\{1,2, \ldots, m\} ; i \neq j\right]
$$

Sujeto a:

$$
\begin{gathered}
d_{i j} \geq L \\
d_{i j}=\sqrt{\left(X_{i}-X_{j}\right)^{2}+\left(Y_{i}-Y_{j}\right)^{2}} \\
L / 2 \leq X_{i}, X_{j} \leq \text { Largo }-L / 2 \\
L / 2 \leq Y_{i}, Y_{j} \leq \text { Ancho }-L / 2
\end{gathered}
$$

Paso 6. El sistema imprime las coordenadas $(X, Y)$ de cada uno de los AP.

\section{Resultados}

A continuación, se presentan los Pseudocódigos 1, 2 y 3 que fueron desarrollados acorde con el modelo propuesto con el fin de optimizar la ubicación de los AP acorde con la metodología descrita anteriormente, minimizando los niveles de interferencia y maximizando el área de cobertura.

Pseudocódigo 1, para optimizar la ubicación de los AP

1. $\mathrm{R} \leftarrow$ input('Ingrese el radio promedio de cobertura: ');

2. Largo $\leftarrow$ input('Ingrese el valor correspondiente al Largo del edificio: ');

3. Ancho $\leftarrow$ input('Ingrese el valor correspondiente al Ancho del edificio: ');

4. $L \leftarrow \operatorname{sqrt}(2) * R$;

5. Area $\leftarrow C=L^{*} L$

6. Area_E $\leftarrow$ Largo*Ancho;

7. $n A P \leftarrow \operatorname{round}($ Area_E/Area_C); // Estimación del número de AP requerido

8. $A \leftarrow[] ;$

9. $b \leftarrow[]$; 
10. $x 0 \leftarrow \operatorname{zeros}\left(1,2^{*} \mathrm{nAP}\right) ; / /$ Vector de condiciones iniciales

11. Aeq $\leftarrow[]$

12. beq $\leftarrow[]$;

// Límites superior e inferior del vector $\mathrm{x}$

13. $X i \leftarrow L / 2$;

14. $X f \leftarrow$ Largo-Xi;

15. $Y i \leftarrow L / 2$;

16. Yf $\leftarrow$ Ancho-Yi;

17. Para i desde 1 hasta $\mathrm{nAP}$

18. $\quad \mathrm{lb}(\mathrm{i}) \leftarrow \mathrm{Xi}$;

19. $\quad l b(i+n A P) \leftarrow Y i$

20. $u b(i) \leftarrow X f$;

21. $u b(i+n A P) \leftarrow Y f ;$

22. Fin Para

// Solución del sistema

23. $[x, f v a l] \leftarrow$ fmincon $(@(x)$ funobj $(x, R, n A P), x 0, A, b, A e q, b e q, l b, u b, @(x)$

restricnl(x,L,nAP));

24. $\operatorname{EjeX} \leftarrow x(1: n A P)$;

25 EjeY $\leftarrow x(n A P+1: 2 * n A P) ;$

26. Resultado $\leftarrow$ [EjeX' EjeY'];

27. Imprimir Resultado

Fuente: Elaboración propia 
Pseudocódigo 2, para la construcción de la función objetivo en el modelo de optimización de la ubicación de los AP

funcion $f \leftarrow$ funobj $(x, R, n A P) / /$ Función objetivo a maximizar

// Rutina para minimizar el área de cobertura

1. $v \leftarrow$ 1:1:nAP; $\quad$ // Vector número de AP

2. $n \leftarrow$ nchoosek $(n A P, 2) ; / /$ Número de enlaces entre AP

3. $\mathrm{Cn} 1 \leftarrow$ nchoosek $(\mathrm{v}, 2) ; / /$ matriz de combinaciones entre enlaces posibles

4. $\mathrm{f} 1 \leftarrow 0$;

5. Para i desde 1 hasta $n$

// Distancia entre APs

6. $d \leftarrow\left((x(\operatorname{Cn} 1(i, 1))-x(C n 1(i, 2)))^{\wedge} 2+(x(\operatorname{Cn} 1(i, 1)+n A P)-x(C n 1(i, 2)+n A P)) \wedge 2\right)^{\wedge} 0.5$;

// Angulo para estimar el área del semiarco de Interferencia

7. Angulo $\leftarrow \operatorname{acos}((d / 2) / R)$;

//Cálculo de área de Interferencia

8. Interf $\leftarrow\left(2^{*} \mathrm{R}^{\wedge} 2\right)^{\star}($ Angulo-(sin(Angulo)* $\cos ($ Angulo $\left.))\right)^{*}$ heaviside $\left(2^{*} \mathrm{R}-\mathrm{d}\right)$;

9. $f 1 \leftarrow f 1+d$; // Sumatoria de interferencias

10. Fin Para

11. $f \leftarrow-f 1 ; \quad$ // Función objetivo a maximizar

Fin funcion

Fuente: Elaboración propia

Pseudocódigo 3, que contiene las restricciones no lineales para el modelo de optimización de la ubicación de los AP

funcion $[c, c e q] \leftarrow \operatorname{restricnl}(x, L, n A P)$

1. ceq $\leftarrow[]$;

2. $R \leftarrow L / s q r t(2)$; 
3. $v \leftarrow 1: 1: n A P ; / /$ Vector número de AP

4. $\mathrm{n} \leftarrow$ nchoosek(nAP,2); // Número de enlaces entre AP

5. $\mathrm{Cn} \leftarrow \mathrm{nchoosek}(\mathrm{v}, 2) ; / /$ Elabora la matriz de combinaciones entre enlaces posibles

6. Para i desde 1 hasta $n$

7. $c(i) \leftarrow-\left((x(C n(i, 1))-x(C n(i, 2)))^{\wedge} 2+(x(C n(i, 1)+n A P)-x(C n(i, 2)+n A P))^{\wedge} 2\right)^{\wedge} 0.5+L$;

8. Fin Para

Fin funcion

Fuente: Elaboración propia

Para evaluar la validez del modelo propuesto, se introducirán en el sistema los parámetros de dos casos y se evaluarán los resultados obtenidos:

Caso 1: En la Figura 4 se presenta el escenario propuesto con los siguientes parámetros: Radio de cobertura 15 , largo y ancho del escenario de prueba $60 \mathrm{~m}$ y $34 \mathrm{~m}$ respectivamente. El resultado obtenido es el siguiente:

Resultado $=$

\author{
10.606623 .3934 \\ 22.395510 .6066 \\ $33.8867 \quad 23.3934$ \\ 49.393410 .6066
}

En este primer caso se observa que para un radio de cobertura de $15 \mathrm{~m}$ y acorde con las dimensiones del edificio, el modelo sugiere que se requiere de al menos 4 Access Point distribuidos tal como se establece en la Figura 5. En la matriz Resultado se imprimen las coordenadas $(X, Y)$ para cada AP, donde la primera columna corresponde a los valores de $\mathrm{X}$ y la segunda los valores de $\mathrm{Y}$. 
Figura 4. Diagrama de cobertura y ubicación de AP - Caso No. 1

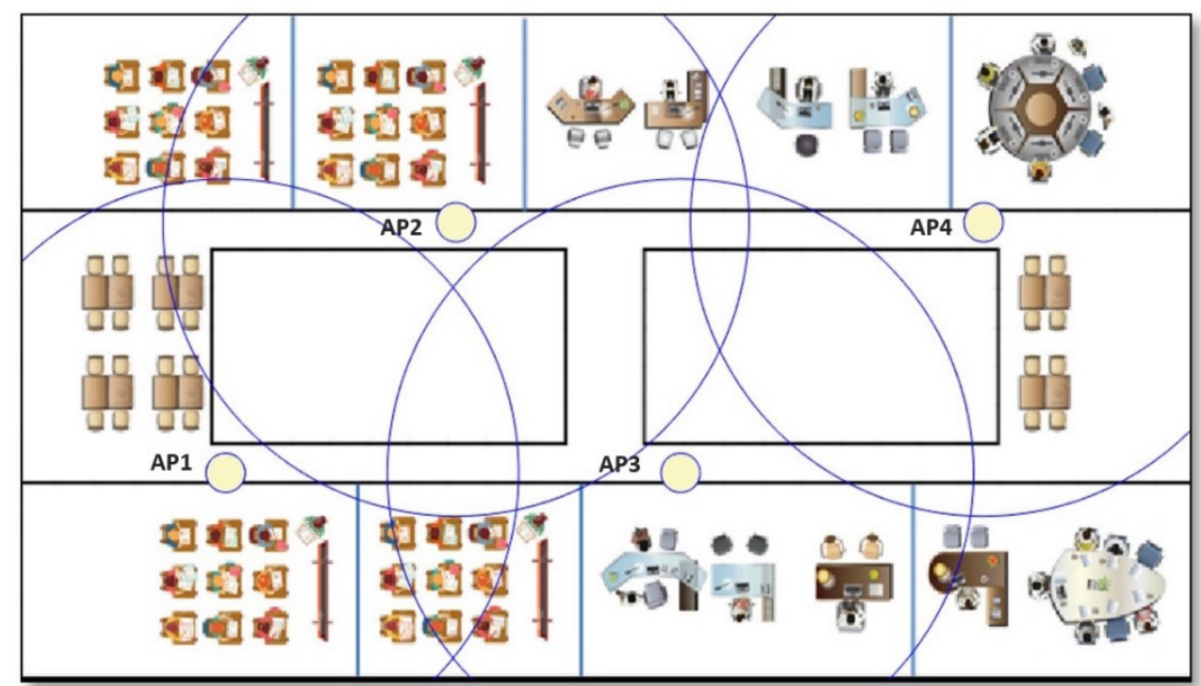

Fuente: Elaboración propia

Caso 2: En la Figura 5 se presenta el escenario propuesto con los siguientes parámetros: Radio de cobertura 20, largo y ancho del escenario de prueba $60 \mathrm{~m}$ y $34 \mathrm{~m}$ respectivamente. El resultado obtenido es el siguiente:

Resultado $=$

$$
\begin{array}{rr}
15.0000 & 8.5000 \\
45.0000 & 25.5000
\end{array}
$$

Figura 5. Diagrama de cobertura y ubicación de AP - Caso No. 2

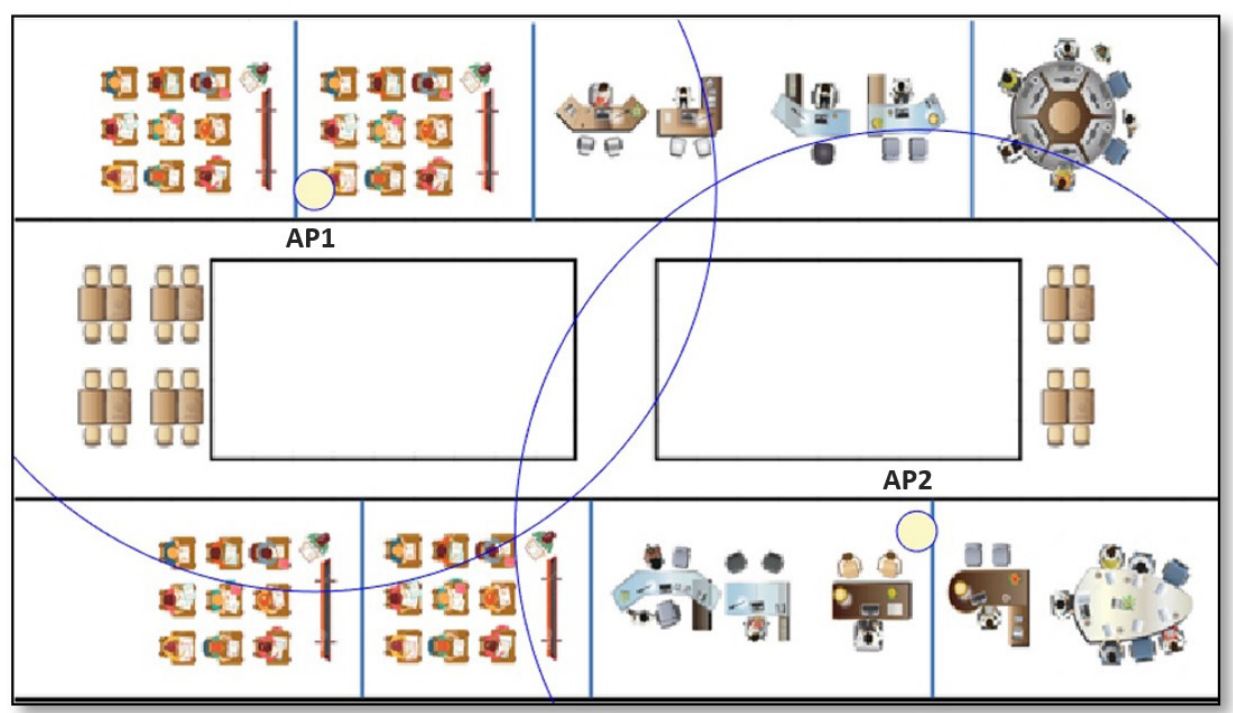

Fuente: Elaboración propia 
En el segundo caso se observa que para un radio de cobertura de $20 \mathrm{~m}$ y acorde con las dimensiones del edificio el sistema sugiere que se requiere de al menos 2 Access Point distribuidos tal como se establece en la Figura 6. En la matriz resultado se imprimen las coordenadas $(X, Y)$ para cada AP, donde la primera columna corresponde a los valores de $\mathrm{X}$ y la segunda los valores de $\mathrm{Y}$.

\section{Conclusiones}

Ante la necesidad de establecer un método que permita optimizar la ubicación de AP en redes WLAN, como estrategia para mejorar los niveles de SINR entre celdas y disminuir los niveles de interferencia entre AP cercanos, se diseñó un modelo de programación no lineal con restricciones claramente definidas y a través del cual es posible maximizar el área de cobertura, minimizar los niveles de interferencia entre celdas, estimar el radio de cobertura de los AP y la probabilidad de corte de la potencia de recepción adecuada con un nivel de sensibilidad entre $-70 \mathrm{dBm}$ y $-80 \mathrm{dBm}$ en escenarios con obstáculos o espacio libre respectivamente, para las bandas de $2.4 \mathrm{GHz}$ y $5 \mathrm{GHz}$. En vista de lo anterior y con base en los resultados obtenidos se pudo evidenciar que el modelo y la metodología propuestos arrojaron excelentes resultados a la hora de establecer las coordenadas cartesianas para la ubicación de los AP en entornos Indoor, brindando una respuesta adecuada a los requerimientos mencionadas anteriormente y soportado bajo un modelo de propagación de atenuación por sombra. Adicionalmente, los algoritmos desarrollados reflejaron una baja complejidad computacional y temporal durante su ejecución, lo cual es muy importante a la hora de analizar y diseñar redes WLAN.

\section{Referencias bibliográficas}

1. N. S. Ravindranath, I. Singh, A. Prasad, and V. S. Rao, Indian journal of science and technology IndJST, vol. 9, no. 26. 2016.

2. A. Kuntal, P. Karmakar, and S. Chakraborty, "Optimization Technique based Localization in IEEE 802.11 WLAN," Recent Adv. Innov. Eng., pp. 1-5, 2014.

3. M. Soleymani, B. Maham, and F. Ashtiani, "Analysis of the downlink saturation throughput of an asymmetric IEEE 802.11n-based WLAN," in 2016 IEEE International Conference on Communications (ICC), 2016, pp. 1-6.

4. F. Juan Carlos Vesga, H. Martha Fabiola Contreras, and W. Harold Esneider Perez, "Optimization of the spectral efficiency in WLAN networks in the $2.4 \mathrm{GHz}$ band under the use of allocation models," Indian J. Sci. Technol., vol. 11, no. 22, pp. 1-13, Jun. 2018.

5. N. S. Ravindranath, I. Singh, A. Prasad, and V. S. Rao, "Performance Evaluation of IEEE 802.11ac and 802.11n using NS3," Indian J. Sci. Technol., vol. 9, no. 26, Jul. 2016.

6. W. A. Syafei, "Implementation of K-Best method for MIMO decoder in WLAN 802.11n," in 2015 2nd International Conference on Information Technology, Computer, and Electrical Engineering (ICITACEE), 2015, pp. 417-421.

7. D. Gong and Y. Yang, "Link-Layer Multicast in Large-Scale 802.11n Wireless LANs with Smart Antennas," IEEE Trans. Comput., vol. 65, no. 7, pp. 2118-2133, Jul. 2016. 
8. M. Abu-Tair and S. N. Bhatti, "Introducing IEEE 802.11ac into existing WLAN deployment scenarios," in 2015 13th International Symposium on Modeling and Optimization in Mobile, Ad Hoc, and Wireless Networks (WiOpt), 2015, pp. 30-35.

9. F. Tramarin, S. Vitturi, M. Luvisotto, and A. Zanella, "On the Use of IEEE 802.11n for Industrial Communications," IEEE Trans. Ind. Informatics, vol. 12, no. 5, pp. 1877-1886, Oct. 2016.

10. S. V. Sangolli and T. Jayavignesh, "TCP Throughput Measurement and Comparison of IEEE 802.11 Legacy, IEEE 802.11n and IEEE 802.11ac Standards," Indian J. Sci. Technol., vol. 8, no. 20, Aug. 2015.

11. H. Basha Pathan, S. Varma P, and S. Rajesh K, "QoSperformance of IEEE 802.11 in MAC and PHY layer using Enhanced OAR Algorithm,” Indian J. Sci. Technol., vol. 10, no. 9, pp. 1-9, Feb. 2017.

12. Y. F. Solahuddin and R. Mardeni, "Indoor empirical path loss prediction model for $2.4 \mathrm{GHz} 802.11 \mathrm{n}$ network," in 2011 IEEE International Conference on Control System, Computing and Engineering, 2011, pp. 12-17.

13. S.-Y. Yeong, W. Al-Salihy, and T.-C. Wan, "Indoor WLAN Monitoring and Planning Using Empirical and Theoretical Propagation Models," in 2010 Second International Conference on Network Applications, Protocols and Services, 2010, pp. 165-169.

14. G. Sun, J. Zhu, R. He, and C. Xiao, “Experimental study of rate-aware scheduling for $802.11 \mathrm{n}$ Wi-Fi network with legacy devices," in 2013 IEEE Global High Tech Congress on Electronics, GHTCE 2013, 2013, pp. 46-49.

15. F. J. Carlos Vesga, H. Martha Fabiola Contreras, and B. Jose Antonio Vesga, “Design of empirical propagation models supported in the Log-Normal Shadowing model for the $2.4 \mathrm{GHz}$ and $5 \mathrm{GHz}$ bands under Indoor environments," Indian J. Sci. Technol., vol. 11, no. 22, pp. 1-18, Jun. 2018.

16. A. del Corte, O. Gutierrez, and J. M. Gomez, "High-accuracy localization based on the dominant rays of ray-tracing over fingerprinting techniques," in Proceedings of the 2012 IEEE International Symposium on Antennas and Propagation, 2012, pp. 1-2.

17. F. J. Carlos Vesga, H. Martha Fabiola Contreras, and W. Harold Esneider Perez, "Use of assignment models as a strategy for channel optimization in the $5 \mathrm{GHz}$ band supported in 802.11ac," Indian J. Sci. Technol., vol. 11, no. 22, pp. 1-17, Jun. 2018.

18. R. Desimone, B. M. Brito, and J. Baston, "Model of indoor signal propagation using log-normal shadowing," in 2015 Long Island Systems, Applications and Technology, 2015, pp. 1-4. 